procedures, responder safety and survival, and emergency communications.

Recommendations: Medical response to disasters is a complex task. First responders perform in a volatile environment to provide maximum care to the needy. Medical first responders should be screened carefully for physical, emotional and mental fitness as well as relativity of medical knowledge background to perform in these less than ideal settings. Medical first responders should synchronize their practice along with the SAR units, or ideally they should be considered as a part of the SAR Team. They should be rigorously trained in different aspects of SAR, as well as disaster and emergency medical care in the field. They should regularly participate in deployment exercises and scenariobased training in cooperation with the other units to maximize the smoothness of interaction between different units, as well as within the same unit with members of different backgrounds.

Conclusion: Turkey lacks the availability of well-trained, disaster, medical first responders. There has been an attempt by the Ministry of Health to address this need. However, forming and training teams to perform according to specified standards and under a unified disaster command has not yet been successful.

Keywords: capabilities; first responders; medical; response; search and rescue (SAR); Turkey

Prehosp Disast Med 2005;20(2):s61-s62

\section{The Journalist's Experience}

Martin Bell, MP

former War Correspondent, BBC

\section{Theme 15: Hot Topic - International Humanitarian Disaster Relief - Tensions and Challenges \\ Chair: Anthony Zwi}

\section{Theme 16: Rural and Remote \\ Emergency Health Care \\ Chairs: Mads Gilbert; James Ferguson}

\section{Theme 17: Landmines}

bairs: Berndt Schneider; Ron Stewart

\section{Free Papers Theme 22: Education-1 Simulations}

Graphic Simulation System for Preparedness of Emergency Department Staff for Mass Casualty Incidents
L. Levi; ${ }^{1}$ D. Bregman ${ }^{2}$
1. Israel
2. Information Systems Department, School of Business Administration, Israel

Problem: Drills are an effective yet resource-consuming technique for improving team preparedness to manage mass-casualty incidents (MCIs). Limited-scale drills, mainly tabletop and communication exercises, are good alternatives, but still harbor deficits, which could be overcome with an interactive simulation system with good graphic interfaces.

Solution: The success of designing such a system, created by students using basic tools, like Visual Basic and MS Access, are described. Basic database terms for the incident types, casualties, treatment requirements, as well as capabilities were used from previous work. The graphic representation included not only the location and movement of casualties and staff, but visualization of treatment phases, as well as problems in management.

The trainee of each of four simultaneous regions actually can treat each victim with choosing possibilities (from small right click window) and requires only minimal typing. The dynamic database can provide online reports that also can be used for the debriefing and benchmarking for the management of that MCI.

Conclusion: Several examples of the system tested in various hospitals will be shown, which gained positive feedback especially for its friendliness and documentation of solutions. Keywords: design; drills; emergency department; mass-casualty incident; simulation

Prebosp Disast Med 2005;20(2):s62

What is a Disaster!? Hospital Disaster Preparedness: Are Hospital Clinical Staff Well-Informed? Does a Mock Disaster Exercise Make a Difference?

B. Bartley, ${ }^{1}$ L. Walsh; ${ }^{2}$ J. Stella, ${ }^{2}$ J. Fisher ${ }^{2}$

1. Barwon Health, Australia

2. Australia

Historically, hospital disaster preparedness has had limited input from clinicians in Australasia. The existence of the "dusty" Disaster Folder is well documented. However, the current climate of terrorism has heightened public awareness of mass casualty incidents. There is a high community expectation that healthcare systems are prepared to cope with any possible event. In Victoria, Australia, there has been a major upsurge in interest in this field among Emergency Medicine clinicians. Other specialties have been less well represented at statewide tabletop exercises and forums.

A statewide, multi-agency "compressed time" mock train crash, "Exercise Kardinia Express", involving 300 patients, took place in North Geelong on Sunday, 10 October 2004. Forty-five moulage patients visited the Geelong emergency department for triage, assessment, and initiation of treatment. Tabletop exercises were performed for the rest of the hospital. As part of the exercise, there was a hot debriefing in the emergency department that day and an enterprise-wide formal debriefing one week later. A one-hour lecture was delivered to the hospital-wide community in the preceding week. Fifty senior medical and nursing staff were surveyed before and after this process to assess their knowledge of the disaster plan and their opinion of hospital disaster preparedness. The pre-intervention data demonstrated some major concerns and deficiencies in knowledge regarding hospital disaster preparedness among both the emergency department and the non-emergency 